\title{
New Markers for Multiple Immunolabeling in FESEM.
}

J. Nebesarova, ${ }^{1,2}$ J.Langhans, ${ }^{1,3}$ J.L.Dean, ${ }^{1,3}$ M.Slouf, ${ }^{4}$ E.Pavlova, ${ }^{4}$ and M.Vancova ${ }^{1}$

${ }^{1}$ Biology Centre of ASCR, Branisovska 31, 37005 Ceske Budejovice, Czech Republic

${ }^{2}$ Faculty of Science, Charles University in Prague, Vinicna 7, 12843 Praha 2, Czech Republic

${ }^{3}$ Faculty of Science, University of South Bohemia, Branisovska 31, 37005 Ceske Budejovice, Czech Republic

${ }^{4}$ Institute of Macromolecular Chemistry, Academy of Sciences of the Czech Republic, Heyrovsky sq. 2, 16206 Praha 6, Czech Republic

A state of art scanning electron microscope equipped with a field emission source (FESEM) is currently reaching such a high resolution which allows to use it broadly for immunolocalisation of cellular structures and components. Immunolabeling methods utilize antigen-specific probes labeled with electron-dense markers/nanoparticles to determine the spatial distribution of the target antigen at high resolution. At present, gold nanoparticles (NPs) are the most common markers in electron microscopy. Backscattered electrons (BSE) are recommended for their detection in FESEM, since the detection in the secondary electron (SE) mode requires particles with a diameter exceeding 30 $\mathrm{nm}$ [1]. The efficiency of immunolabeling depends in addition to other on the size of markers which are most often in the range $5-15 \mathrm{~nm}$. In order to detect such small particles, the most efficient BSE detector must be used.

If there is need to locate more antigens simultaneously the only choice is to use gold NPs of different sizes that can be distinguished in FESEM. Practically it is possible to use Au NPs in the sizes of 5 and $15 \mathrm{~nm}$, which limits the possibility of simultaneous multiple labeling only for two antigens. The aim of this study was to find other colloidal metal NPs suitable as markers and test them for their stability under the electron beam, their ability to conjugate them to antibodies and their distinctness from the gold NPs.

Based on the testing, palladium NPs were selected as the most suitable for multiple immunolabeling in FESEM, because they met the above mentioned conditions [2]. At first we measured the size distribution of Au and Pd NPs in FESEM using the signal of BSE detected with the improved Autrata YAG detector [3]. The measurement was influenced by a number of factors, e.g. accelerating voltage, size and chemical composition of NPs, magnification as well as image processing. The use of BSE imaging for the discrimination of NPs of the same metal with different diameters was possible on condition that the difference between the diameters was at least $10 \mathrm{~nm} . \mathrm{Pd}$ NPs were less stable under the primary electron beam and yielded less signal in comparison with Au NPs. Therefore, the smallest 6nm Pd NPs could not be reliably detected, while the Au NPs of the same diameter were still easily observed and analysed [4].

In the next step we wanted to determine whether it would be possible to distinguish Au and Pd NPs under different BSE signal levels. Figure 1 shows the results of multiple immunolocalisation of fucosylated glycans in secretory granules of salivary glands isolated from females Ixodes ricinus by means of $10 \mathrm{~nm}$ Pd and $10 \mathrm{~nm}$ Au NPs conjugated with streptavidin. Pd and Au NPs were not possible to distinguish in TEM and SE mode of FESEM. However BSE imaging using of Autrata 
YAG detector in combination with sufficient high magnification permitted to distinguish them due to a difference in the BSE signal strength.

\section{References}

[1] M. Mueller et al., in Immunogold labelling in cell biology (Eds.: A.J. Verkleij, J.L.M.

Leunissen), CRC Press Inc., Boca Raton, Florida (1989) 199-216.

[2] M.Vancova et al., Microsc. Microanal. (2010) in revision.

[3] P. Walther et al., Scanning Microsc. 5(2) (1991) 301-310.

[4] J.Nebesarova et al., Ultramicroscopy (2011) in revision.

[5] The authors acknowledge the financial support from grant projects of Academy of

Sciences of the Czech Republic (KAN200520704, Z60220518) and Grant Agency of the Czech Republic (P205/10/0348).

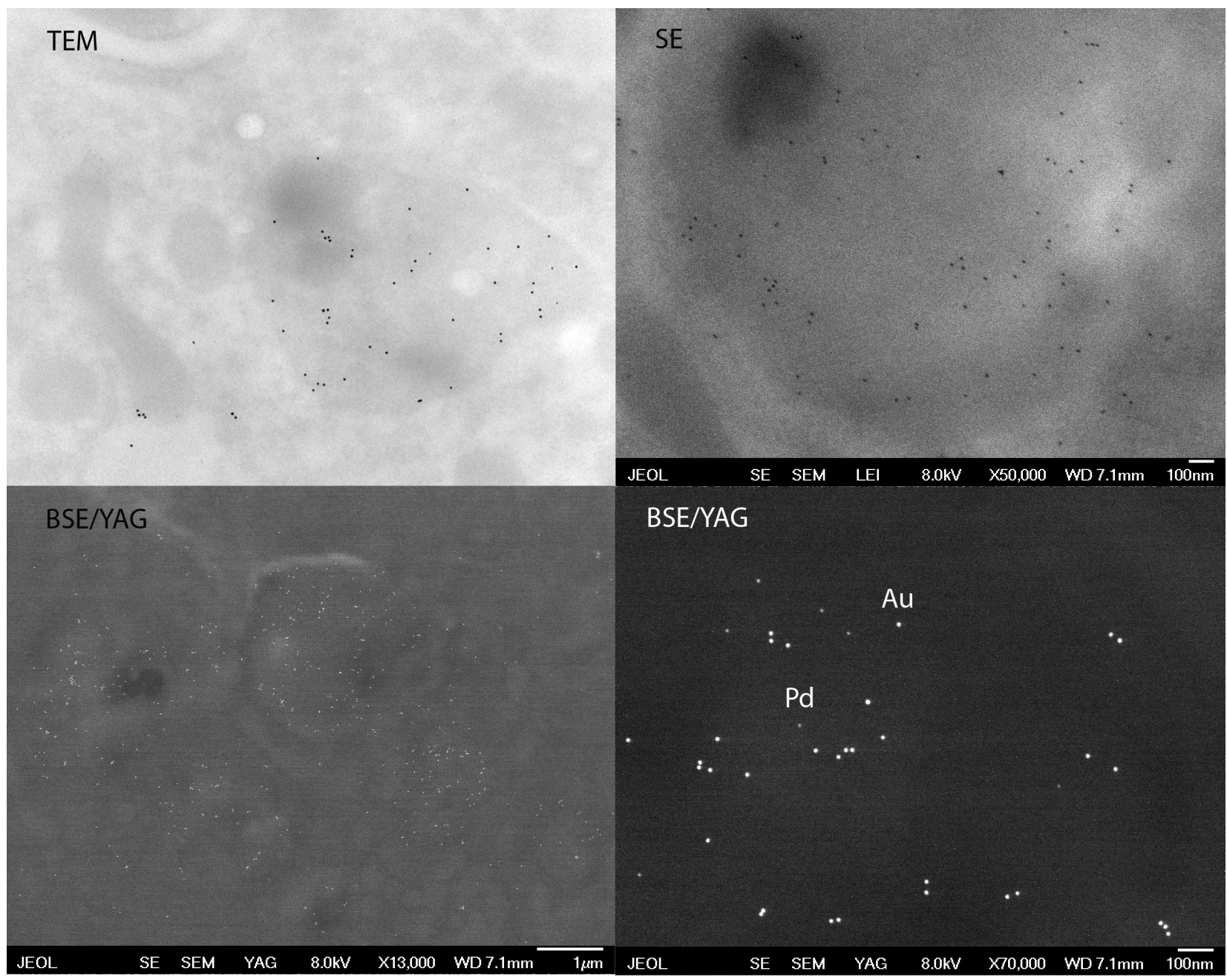

FIG 1. Multiple immunolabeling of fucosylated glycans in secretory granules of salivary glands isolated from females Ixodes ricinus in TEM and FESEM working in SE and BSE mode. $10 \mathrm{~nm}$ Pd and $15 \mathrm{~nm}$ Au nanoparticles conjugated with streptavidin was used as markers. 